\title{
Evaluation of the Effect of a Mobile Phone Application on Treatment Success and Drug Adherence in Hypertensive Patients
}

\author{
Mert Kaşkal $^{*}$, Selçuk Şen ${ }^{2}$ and Yağız Üresin ${ }^{2}$ \\ ${ }^{1}$ Department of Medical Pharmacology, Marmara University, School of Medicine, Turkey \\ ${ }^{2}$ Department of Medical Pharmacology, Istanbul Faculty of Medicine, Istanbul University, Turkey \\ *Corresponding author: Mert Kaşkal, Department of Medical Pharmacology, Marmara University, School of Medicine, 34668, \\ Maltepe, Istanbul, Turkey
}

\section{ARTICLE INFO}

Received: 慧 October 30, 2021

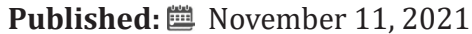

Citation: Mert Kaşkal, Selçuk Şen, Yağız Üresin. Evaluation of the Effect of a Mobile Phone Application on Treatment Success and Drug Adherence in Hypertensive Patients. Biomed J Sci \& Tech Res 39(5)2021. BJSTR. MS.ID.006373.

Keywords: Hypertension; Drug Adherence; Mobile Phone Application; Blood Pressure

\section{ABSTRACT}

Objective: One of the most important factors affecting the prognosis of hypertension is the patients' adherence to drug therapy. In this study, it was aimed to evaluate the improvement in drug adherence in hypertensive patients after using a mobile phone application.

Patients and Methods: In this single-centered study fifty patients with hypertension were recruited. All the patients used the Medisafe ${ }^{\circledR}$ mobile phone application for 8 weeks. The application is intended to increase drug adherence of patients and provide better blood pressure control. The differences in systolic blood pressure (SBP) and diastolic blood pressure (DBP) levels from the baseline and the levels at the end of the study were compared. The drug adherence was evaluated using the 8-question Morisky Drug Adherence Scale (MMAS-8) at baseline and at the end of the study.

Results: The mobile application utilization provided a significant decrease in both the mean SBP levels by $6.2 \mathrm{mmHg}(\mathrm{p}<0,001)$ and in the mean DBP levels by $2.4 \mathrm{mmHg}$ $(\mathrm{p}=0.035)$ Additionally, the mobile application also positively and significantly increased the drug adherence of the patients (mean baseline score: 5.54 vs mean end of the study score:6.3 and $\mathrm{p}=0,001)$. In the subgroup analysis of the study demonstrated that in addition to an increase in patients drug adherence, the use of the mobile application provided a significant decrease in the mean SBP levels by $6.6 \mathrm{mmHg}(\mathrm{p}=0.016)$ in geriatric patients but the decrease in DBP did not significantly change $(p>0.05)$. In geriatric patients, the mean difference between drug adherence scores baseline and after application was found to be significant $(\mathrm{p}=0.006)$. With the use of mobile application, patients with polypharmacy had decreased mean SBP and DBP levels, and an increased mean MMAS-8 score of 5.07 to 5.85 ( $\mathrm{p}=0.009)$.

Conclusion: The present pilot study demonstrated that mobile application use in patients with hypertension may increase the treatment success and the patients drug adherence. The beneficial effects of mobile applications in the treatment of hypertension and other common chronic diseases should be evaluated in further larger clinical trials.

\section{Introduction}

Hypertension is one of the leading causes of cardiovascular mortality and morbidity and the prevalence increasing with age. Uncontrolled hypertension is highly associated with cardiovascular and cerebrovascular diseases including myocardial infarction heart failure, stroke, and dementia [1]. It is estimated that the prevalence of hypertension in patients above 60 years of age is approximately 
$60 \%$ [2]. One of the main adversities concerning the control of hypertension is the inappropriate use of drugs. Drug adherence is about $50 \%$ in hypertension patients according to the literature. Adherence is the adaptation of a person to lifestyle changes, diet, and medication given by the healthcare professional [3]. The level of compliance with the drug treatment is called drug adherence. A low level of drug adherence is one of the important challenges for the management of chronic diseases. Sociodemographic factors such as age, gender, and education levels contribute to drug adherence.

It was shown that the complications of chronic diseases are more frequently observed in elderly patients who have low drug adherence [4]. It is a fact that the drug adherence is not at a satisfactory level globally [5]. A poorly organized healthcare system, poor access to medication and inability to provide a rapid recovery from drugs especially in chronic diseases, and a lack of information about the disease may lower drug adherence. On the other hand, if good communication between patients and physician is maintained, drug adherence increases. Therefore, patient involvement in the management of chronic diseases should not be ignored. There is a need to increase the awareness and the involvement of patients in the treatment to improve drug adherence. The widespread use of new approaches and technological tools may provide an opportunity. In hypertensive patients, a high level of drug adherence is important to maintain optimal levels of blood pressure and to prevent complications.

One of the main reasons for drug-resistant hypertension is the lack of drug adherence and it is shown that only $50 \%$ of hypertensive patients take the prescribed drugs appropriately [6]. In cases where higher drug adherence levels are achieved, it is stated that risk of stroke is reduced by $35-40 \%$ and the risk of myocardial infarction is reduced by $20-25 \%[7,8]$. In recent years the use of mobile applications in the treatment of chronic diseases such as hypertension has increased considerably. More than 100 mobile applications for hypertension are available from different sources. In studies in which the effect of mobile phone applications on hypertension were evaluated, it has been shown that the use of applications increased patient-physician coordination, increased patient's awareness of hypertension, and ensured the patient take more responsibility for their hypertension $[9,10]$. The Morisky Medication Drug Adherence Scale-8(MMAS-8) is a survey including eight questions that are commonly used to evaluate drug adherence rates in hypertensive patients. It is shown that MMAS- 8 is a useful tool for detecting low drug adherence in hypertensive patients [11]. In this study, we aimed to investigate the change of drug adherence rates in hypertensive patients after the use of mobile phone applications for the management of hypertension.

\section{Methods \\ Study Population}

Fifty patients with hypertension who were age $\geq 18$ and under treatment with anti-hypertensive medication for at least three months and presented to Istanbul Medical Faculty Department of Clinical Pharmacology Polyclinic were included in this study. The study was approved by the Istanbul Medical Faculty Clinical Research Ethics Committee and enrolled participants gave their informed consent.

\section{Blood Pressure Measurement}

Blood pressure measurements were taken after resting for a minimum of 5 minutes in a sitting position, measured using a clinical-trial-use approved, validated, and calibrated oscillatory blood pressure measurement device (Omron 7051T, Kyoto, Japan). The patients did not take caffeinated drinks or smoked cigarettes 30 minutes before measurement. Two measurements were taken in 5-minute intervals and blood pressure levels were recorded as the average of those 2 measurements. Blood pressure measurements were done in office conditions at the beginning and at the end of 8 weeks. The baseline blood pressure and the blood pressure after 8 weeks of application use were considered. The blood pressure measurement and regulations were taken according to guideline of 2018 ESC/ESH Guidelines for the management of arterial hypertension.

\section{Evaluation of Drug Adherence}

Blood pressure measurements were done and patients were asked questions from MMAS-8. According to the answers, patients were classified as low adherers, medium adherers, and high adherers. Patients who scored less than 6 points were categorized as low adherers, patients who scored between 6-8 points were categorized as medium adherers and patients who scored 8 points were categorized as high adherers according to the MMAS- 8 scale [11]. After the questionnaire, the Medisafe ${ }^{\circledR}$ program which is a free application available at the Apple Store and Google Play Store had been downloaded on the mobile phones of the patients. The Medisafe ${ }^{\circledR}$ application was chosen because it has an easy interface to use, and it is available for free. The name of the medication, the medication administering time had already been recorded into the Medisafe ${ }^{\circledR}$ program by the physician. After eight weeks, blood pressure measurements were taken and drug adherence rates were assessed by MMAS-8 scale.

\section{Statistical Analysis}

Statistical analyses were performed using IBM's Statistical Package for the Social Sciences (SPSS) Windows version 21.0. The 
distribution of variables was tested using the Shapiro-Wilk test. For variables with normal distribution, paired t-test and Pearson correlation analyses were performed. For variables without normal distribution, the Wilcoxon Signed rank and Spearman's rho correlation analysis were conducted. Pearson's Chi-square test was used to examine the differences between categorical variables. The results were considered significant if $\mathrm{p}<0.05$.

\section{Results}

\section{Participants}

Table 1: The number of medications of the patients.

\begin{tabular}{|c|c|}
\hline Number of drugs & Number of patients \\
\hline Monotherapy & 12 \\
\hline Two drug combinations & 13 \\
\hline Three drug combinations & 11 \\
\hline Four drug combinations & 9 \\
\hline More than four drug combinations & 5 \\
\hline Total & 50 patients \\
\hline
\end{tabular}

Fifty patients (26 male/24 female) with the mean age 60.16 years (range 33-75 years) were consecutively enrolled in the study. The mean age of the patients was 60.16 years, ranging between 33-75 years. Patients taking four or more medications a day were considered as patients with polypharmacy. The average number of medications taken in patients with polypharmacy was 4.5. Patients who were taking less than four medications a day were considered as patients with non-polypharmacy. The average number of medications taken in patients with non-polypharmacy was 1.97. The number of patients with polypharmacy was thirty-six and patients with non-polypharmacy was fourteen. Eighteen patients were defined as geriatric patients (aged $\geq 65$ ), the mean age of the geriatric patients was 68.8. Thirty-two patients were non-geriatric patients (aged $<65$ ), the mean age of the non-geriatric patients was 55.4. The number of medications given are indicated in Table 1.

\section{Drug Adherence}

The drug adherence of patients was evaluated with MMAS-8. At baseline, twenty-five patients were found low adherent (\%50), twenty patients were found medium adherent (\%40), and five patients high adherent (\%10). The MMAS-8 score average was found to be 5.54. After application, the patients' drug adherence was evaluated using MMAS-8. After application, fourteen patients were found low adherent (\%28), thirty-one patients were found medium adherent (\%62), and five patients high adherent (\%10). The MMAS-8 score average was found to be 6.3. After application twenty-two patients' drug adherence rates were improved. The change in MMAS-8 scores at baseline and after application was found significant $(\mathrm{p}<0,001)$. In patients with polypharmacy, nine patients were found low adherent (\%64\%) and five patients were found medium adherent (36\%). In non-polypharmacy patients, sixteen patients were found low adherent (44\%), fifteen patients were found medium adherent (42\%) and five patients were found high adherent (14\%).

The MMAS-8 score average was found to be 5.07 in the polypharmacy group and 5.72 in the non-polypharmacy group. Non-polypharmacy patients were found more adherent to the drug regimen and the difference in the MMAS-8 scores was found statistically significant $(\mathrm{p}=0.015)$. After application, the average MMAS-8 score in polypharmacy patients was 5.85 ( $\mathrm{p}=0.009)$. In non-geriatric patients, the average MMAS-8 score was 5.71 . In geriatric patients, and the baseline and the after application MMAS8 scores were 5.22 and 6.11 respectively $(p=0.006)$. Additionally, there was no significant difference found in the MMAS-8 score average between women and men ( $p>0.05$ ) (Figure 1).

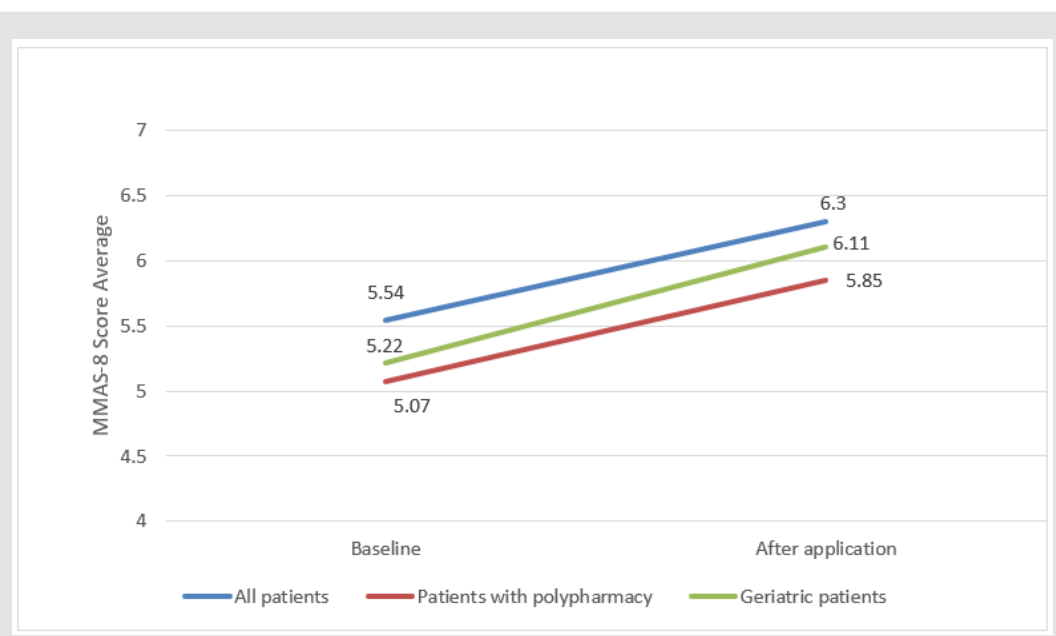

Figure 1: The differences between the baseline MMAS-8 score averages of patients before and after 8 weeks 


\section{Blood Pressure}

The mean systolic blood pressure (SBP) at baseline and after application were $136.4 \pm 14.52,130.2 \pm 13.22 \mathrm{mmHg}$ respectively $(\mathrm{p}<0.001)$. The mean diastolic blood pressure (DBP) at baseline and after application were $81.1 \pm 9.65,78.8 \pm 8.57 \mathrm{mmHg}$ respectively $(p=0.035)$ (Figure 2). We evaluated the blood pressure levels and MMAS-8 score of patients with polypharmacy before and after the use of mobile phone applications. The average SBP levels of patients with polypharmacy baseline and after application were $129.3 \pm 16.5,123.6 \pm 11.02 \mathrm{mmHg}$ respectively $(p=0.045)$. A significant difference was not detected for the difference in DBP of patients with polypharmacy before and after the application. The decrease in SBP of geriatric patients after mobile phone application was found statistically significant $(p=0.016)$. However, the change in the DBP of geriatric patients was found statistically insignificant $(\mathrm{p}=0.178)$. In addition, an inverse correlation was found between the change in the MMAS-8 score of patients and the change in SBP ( $\mathrm{r}:-0.554 ; \mathrm{p}<0.001)$ and in DBP ( $\mathrm{r}:-0.484 ; \mathrm{p}<0.001)$.

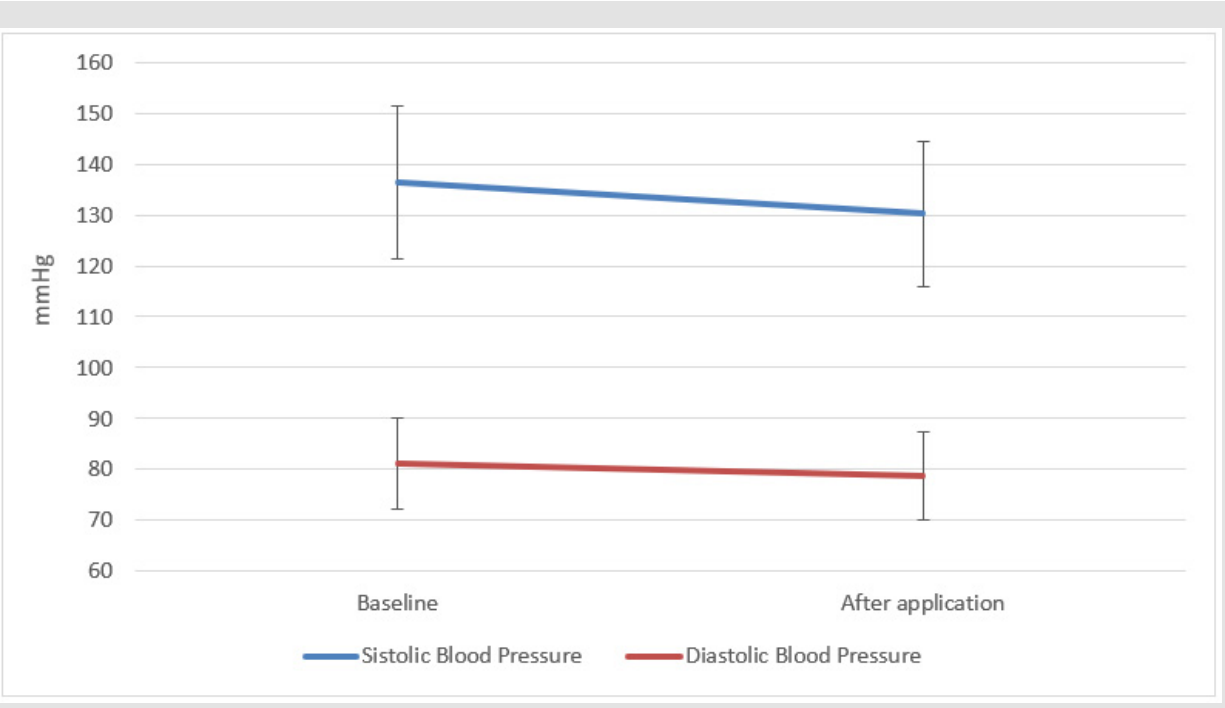

Figure 2: The differences between the baseline blood pressure of patients and after application.

There was no significant difference found between the SBP and DBP levels between women and men ( $p>0.05)$. At baseline, 21 patients had uncontrolled hypertension and 29 patients had controlled hypertension. After application, 15 patients had uncontrolled hypertension and 35 patients had controlled hypertension. 7 patients moved from the uncontrolled to the controlled group whereas 1 patient moved from the controlled group to the uncontrolled group (Figures $3 \& 4$ ).

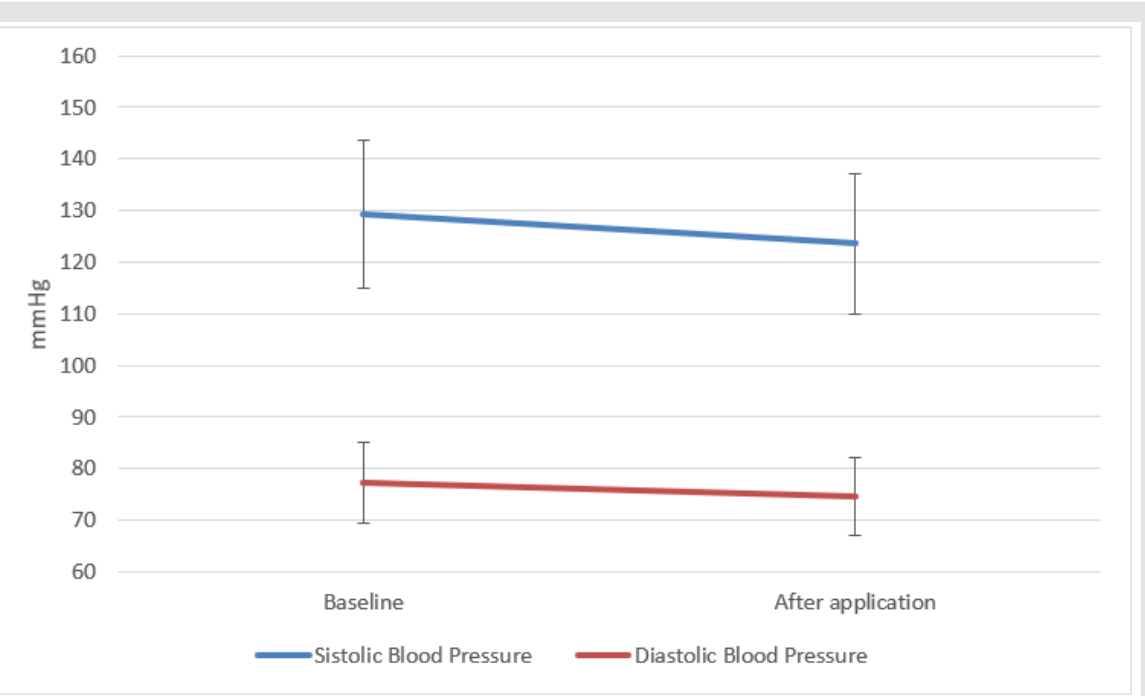

Figure 3: The differences between the blood pressure of patients with polypharmacy baseline and after application. 


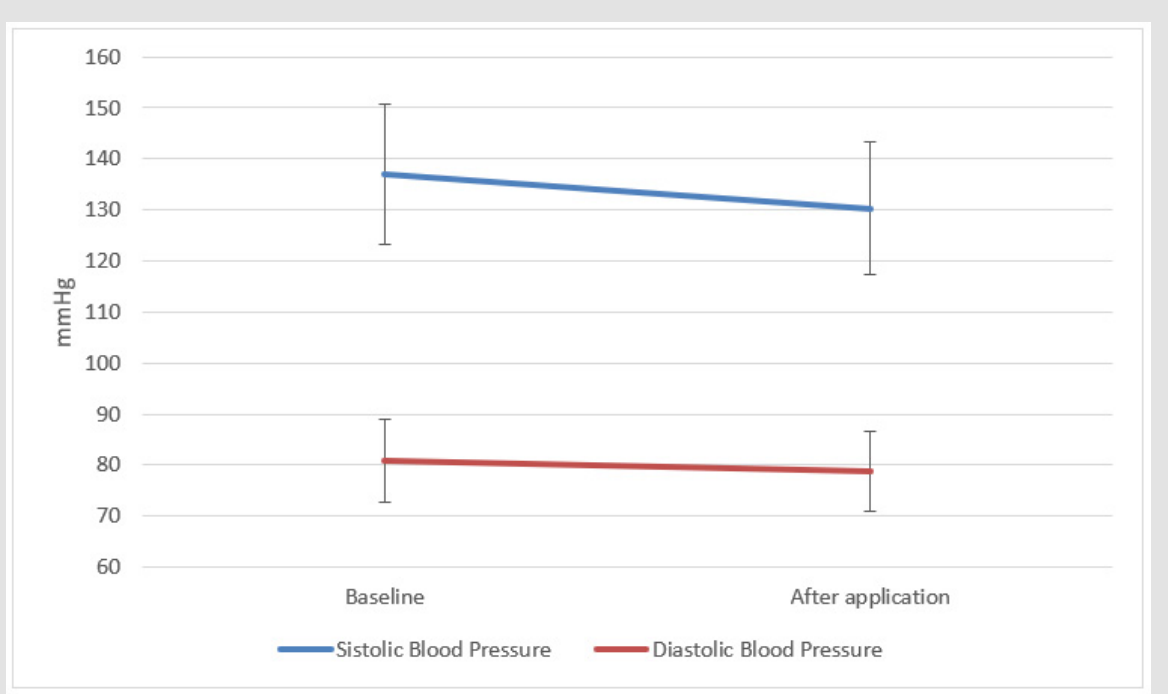

Figure 4: The differences between the baseline and after application blood pressure values in geriatric patients.

\section{Opinion of Patients about Application}

After the use of the application for 8 weeks, patients were asked two questions to assess their opinions about the application. The first question patients were asked was whether they thought the application made it easier for them to take their medication. 33 patients out of 50 reported that the application facilitated their medicine intake and that they would continue using the Medisafe ${ }^{\circledR}$ application. The second question addressed whether the application facilitated the patients' blood pressure management. 26 patients stated that the use of the Medisafe ${ }^{\circledR}$ application had a benefit for the management of their hypertension.

\section{Discussion}

The prevalence of hypertension has risen in recent years due to the average lifespan increase in the general population. The control of hypertension has a great importance in reducing cardiovascular and cerebrovascular diseases, thus reducing the additional morbidity and mortality. Also, cognitive functions may decline with age and cognitive impairment may be seen. It is common to see lower drug adherence rates in the aging population because of possible cognitive impairment. One of the most important points in the management of hypertension is to maintain high drug adherence. Different approaches have been developed to facilitate chronic drug use. One of these approaches is the use of mobile phone applications. In this study, we analyzed the effects of publicly available mobile phone application usage on SBP, DBP and drug adherence. With the introduction of smartphones into our lives, health applications that can be used on these mobile phones have also emerged. Health applications have features such as recording patients' blood tests, appointment reminders, recording blood pressure values, recording unusual symptom, and transmitting these records to doctors instantly and other special features.
With the use of these mobile phone health applications, it is thought that awareness about the diseases has increased, and the management of these diseases has improved, especially in people with chronic diseases. These kinds of approaches increase patients' involvement in the management of chronic diseases. In our study, we found significant reduction in the SBP and DBP in patients after application use. Additionally, average MMAS-8 scores of the patients increased after 8 weeks of application use which is a sign that mobile application use increases drug adherence. With mobile application use, remarkable results were found especially in the geriatric patients who were not familiar with technological interventions. Since our study was a pilot study, there was no control group. Nevertheless, more clinical studies in larger patient groups should be done to verify the positive results found in our study. There are other studies on chronic diseases using mobile phone applications. The effect of mobile phone application use on hypertensive patients was evaluated, the results concluding that the use of mobile phone application did not provide an improvement in SBP but increased the drug adherence [12].

In a study on patients with heart failure, a mobile phone application was used to record the health data of these patients, and the information was shared with the patients' physicians [13]. Thus, it was aimed to increase the awareness of patients about heart failure and to decrease hospitalization. In another study carried out on hypertensive patients, the blood pressure values and drug adherence of the patients were evaluated after using a mobile phone application, and a significant decrease in blood pressure values and an increase in drug adherence were found [8]. In our study, we mainly evaluated the blood pressure and drug adherence rates of patients at baseline and after application use. The decrease in SBP after the use of mobile phone applications was found statistically significant $(\mathrm{p}<0.001)$. The decrease in DBP was found 
less significant $(\mathrm{p}=0.035)$. It was stated that an average decrease of $10 \mathrm{mmHg}$ in SBP and an average decrease of $5 \mathrm{mmHg}$ in DBP, reduces morbidity and mortality by preventing cardiovascular and cerebrovascular diseases [14,15]. Considering this evidence, the use of application would be valuable in preventing cardiovascular and cerebrovascular morbidity and mortality.

After application use, the overall MMAS-8 score average and patients drug adherence rates were increased. The rise in the MMAS-8 score average was found statistically significant after application use $(p=0.001)$. Recent studies also showed that mobile phone applications that provide drug reminders and help patients to record their health data regularly, increase drug adherence especially in patients with chronic diseases and using multiple drugs $[16,17]$. It is thought that decreased cognitive functions with aging may cause cognitive decline and this may decrease drug adherence in geriatric patients. For this reason, we have evaluated the drug adherence of patients over the age of 65 who participated in our study before and after the use of mobile phone applications. An increase in MMAS-8 score after application use was found statistically significant in geriatric patients $(\mathrm{p}=0.006)$. Additionally, the decrease in SBP in geriatric patients was found significant $(p=0.016)$. The decrease in DBP was found statistically insignificant $(p=0.178)$. The use of multiple drugs may be one of the factors contributing to low drug adherence.

It was shown that an increasing number of drugs may reduce the drug adherence of patients $[18,19]$. In our study, patients taking four or more medications a day were polypharmacy patients. The MMAS-8 score of non-polypharmacy patients compared to polypharmacy patients was found higher and statistically significant $(p=0.015)$. The decrease in SBP values of polypharmacy patients before and after application use was found to be significantly lower $(\mathrm{p}=0.045)$. The decrease in DBP was not found to be statistically significant ( $p>0.05$ ). The increase in the MMAS-8 score of polypharmacy patients after mobile phone application was found significant $(\mathrm{p}=0.009)$

\section{Conclusion}

The use of mobile phone application increased drug adherence rates and maintained better control of blood pressure. In order to reach a more definite conclusion there is need for further clinical studies on larger groups of patients evaluating the benefit of mobile phone applications on drug adherence and blood pressure. In parallel to technologic improvement the use of remote monitoring systems with more detailed features will provide better control for the patients and should be encouraged.

\section{Limitations}

The results of this present study may support beneficial effects of mobile phone application use. The present pilot study had a relatively small sample size and did not have a control group. The results of this study are beneficial to the design further larger clinical studies. Further larger clinical studies including control groups should be performed to reach a definitive conclusion

\section{Acknowledgement}

This study was presented as an oral presentation at the $6^{\text {th }}$ International Scientific Congress (UTSAK) held online between 1011 April 2021.

\section{Conflict of Interest}

The authors declared that they no conflict of interest.

\section{References}

1. ESC/ESH Guidelines for the management of arterial hypertension 2018.

2. Chow CK, Teo KK, Rangarajan S, Islam S, Gupta R, et al. (2013) Prevalence, awareness, treatment, and control of hypertension in rural and urban communities in high-, middle-, and low-income countries. JAMA 310 959-968.

3. Sabate E (2001) WHO Adherence Meeting Report. Geneva, World Health Organization.

4. Jernigan JA (1948) Update on drugs and the elderly. American Family Physician 29: 238-247.

5. Burnier M (2017) Drug adherence in hypertension. Pharmacol Res 125(Pt B): 142-149.

6. De Oliveira-Filho AD, Costa FA, Neves SJ, Junior DPL, Morisky DE (2014) Pseudoresistant hypertension due to poor medication adherence. Int J Cardiol 172(2): 309-310.

7. Chobanian AV, Bakris G, Black HR, Cushman WC, Green LA, et al. (2003) Seventh report of the Joint National Committee on Prevention, Detection, Evaluation, and Treatment of High Blood Pressure. Hypertension 42: 1206-1252.

8. EM Contreras, Rivero SM, Garcia ER, Ramos LLG, Vilas JCP, et al. (2019) Specific hypertension smartphone application to improve medication adherence in hypertension: a cluster-randomized trial. Current Medical Research and Opinion 35(1): 167-173.

9. Moore JO, Marshall MA, Judge DC, Moss FH, Gilroy SJ, et al. (2014) Technology-supported apprenticeship in the management of hypertension: a randomized controlled trial. J Clin Outcomes Manag 21(3): 110-122.

10. Thakkar J, Kurup R, Laba TL, Santo K, Thiagalingam A, et al. (2016) Mobile telephone text messaging for medication adherence in chronic disease: a meta-analysis. JAMA Intern Med 176(3): 340-349.

11. Morisky DE, Ang A, Krousel-Wood M, Ward HJ (2008) Predictive validity of a medication adherence measure in an outpatient setting. J Clin Hypertens(Greenwich) 10(5): 348-354.

12. Morawski K, Ghazinouri R, Krumme A, Lauffenburger JC, Lu Z, et al. (2018) Association of a Smartphone Application with Medication Adherence and Blood Pressure Control: The MedISAFE-BP Randomized Clinical Trial. JAMA Intern Med 178(6): 802-809.

13. Arulnathan A, Vaaheesan S, Denecke K (2019) A Mobile Application for Self-Monitoring for Patients with Heart Failure. Stud Health Technol Inform 259: 113-116.

14. Ettehad D, Emdin CA, Kiran A, Anderson SG, Callender T, et al. (2016) Blood pressure lowering for prevention of cardiovascular disease and death: a systematic review and meta-analysis. Lancet 387: 957-967. 
15. Thomopoulos C, Parati G, Zanchetti A (2014) Effects of blood pressure lowering on outcome incidence in hypertension. 1. Overview, metaanalyses, and metaregression analyses of randomized trials. J Hypertens 32: 2285-2295.

16. Martin CA, Upvall MJ (2016) A Mobile Phone HIV Medication Adherence Intervention: Acceptability and Feasibility Study. J Assoc Nurses AIDS Care 27(6): 804-816

17. Becker S, Kribben A, Meister S, Diamantidis CJ, Unger N, et al. (2013) User profiles of a smartphone application to support drug adherence-experiences from the iNephro project. PLoS One 8(10): e78547.

\section{ISSN: 2574-1241}

DOI: 10.26717/BJSTR.2021.39.006373

Mert Kaşkal. Biomed J Sci \& Tech Res

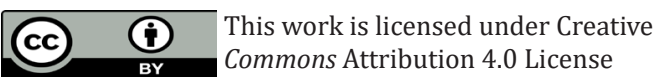

Submission Link: https://biomedres.us/submit-manuscript.php
18. Ulley J, Harrop D, Ali A, Alton S, Davis SF (2019) Deprescribing interventions and their impact on medication adherence in communitydwelling older adults with polypharmacy: a systematic review. BMC Geriatr 19(1): 15.

19. Pasina L, Brucato AL, Falcone C, Cucchi E, Bresciani A, et al. (2014) Medication non-adherence among elderly patients newly discharged and receiving polypharmacy. Drugs Aging 31(4): 283-289.

$\begin{array}{ll}\text { BIOMEDICAL } & \text { Assets of Publishing with us } \\ \text { RESEARCHES } & \text { - Global archiving of articles } \\ \text { - Immediate, unrestricted online access }\end{array}$

